\title{
Comparing Downside Risk Measures for Heavy Tailed Distributions*
}

\author{
Jón Daníelsson \\ Bjørn N. Jorgensen \\ London School of Economics \\ Columbia Business School \\ Mandira Sarma \\ Indian Statistical Institute, Delhi \\ EURANDOM, Eindhoven University of Technology \\ Casper G. de Vries \\ Erasmus University Rotterdam \\ Tinbergen Institute \\ EURANDOM
}

December 2005

\begin{abstract}
Using regular variation to define heavy tailed distributions, we show that prominent downside risk measures produce similar and consistent ranking of heavy tailed risk. Thus regardless of the particular risk measure being used, assets will be ranked in a similar and consistent manner for heavy tailed assets.
\end{abstract}

KEY WORDS: downside risk measures, heavy tailed distribution, regular variation

JEL Classification: D81, G11

\footnotetext{
${ }^{*}$ We thank the referee for suggesting Corollary 1. Danielsson acknowledges the financial support of the EPSRC grant no. GR/S83975/01. His papers can be downloaded from www. RiskResearch.org.
} 


\section{Introduction}

Dhaene et al. (2003) define downside risk measures as measures of the "distance" between a risky situation and the corresponding risk-free situation when only unfavourable discrepancies contribute to the "risk". The early literature on downside risk measures dates back to the "safety first" rule of Roy (1952). Subsequently lower partial moments were proposed which defined risk as the probability weighted function of the deviations below a target return (Bawa, 1975; Fishburn, 1977). There is a renewed interest in downside risk measures due to the prominence of concepts like Value-atRisk (VaR) and Expected Shortfall (ES) for financial risk management and prudential regulation.

Here we consider several important downside risk measures, viz., lower partial moments (of second, first and zeroth orders), VaR and ES for heavy tailed asset returns. Using the notion of "regular variation" to define heavy tails, we provide approximations of the risk measures in the tail region. Further, we show analytically that the heavy tailed feature induces similar asset rankings regardless of the particular risk measure being used.

\section{Heavy Tailed Distribution and Downside Risk Measures}

Many empirical studies have established that asset returns exhibit heavy tails (see e.g. Mandelbrot, 1963; Engle, 1982; Jansen and de Vries, 1991; Pagan, 1996). In these studies heavy tailed distributions are often defined in terms of higher than normal kurtosis. However, higher than normal kurtosis is not a sufficient condition for heavy tails. Kurtosis only captures the probability mass of the distribution at the centre relative to the tails. Indeed, it is straightforward to construct a distribution with truncated tails, and hence thin tails, which exhibits high kurtosis. In this paper we define a heavy tailed distribution as one characterised by the failure of the moments of order $m(>0)$ or higher. Such distributions have tails that exhibit a power type behaviour like the Pareto distribution, as is commonly observed in finance. Such tail behaviour can be mathematically defined by using the notion of "regular variation", as defined below. ${ }^{1}$

\footnotetext{
${ }^{1}$ For an encyclopaedic treatment of regular variation, see Bingham et al. (1987); Resnick (1987).
} 


\subsection{Regular Variation}

A cumulative density function (cdf) $F(x)$ varies regularly at minus infinity with tail index $\alpha>0$ if

$$
\lim _{t \rightarrow \infty} \frac{F(-t x)}{F(-t)}=x^{-\alpha} \quad \forall x>0
$$

This implies that to a first order approximation, all distributions with regular variation have a tail similar to the Pareto distribution:

$$
F(-x)=A x^{-\alpha}[1+o(1)], x>0, \text { for } \alpha>0 \text { and } A>0
$$

For distributions with regularly varying tails, moments of order $m>\alpha$ are unbounded and therefore these distributions display heavy tailed behaviour. The power $\alpha$ is called the tail index and determines the number of bounded moments; $A$ is the scale coefficient. It is easily verified that Student-t distributions vary regularly at infinity with degrees of freedom equal to the tail index and satisfy the above approximation. Likewise, the stationary distribution of the popular $\operatorname{GARCH}(1,1)$ process has regularly varying tails, see de Haan et al. (1989).

\section{Downside Risk Measures for Heavy Tailed Distributions}

We consider the following downside risk measures.

Second Lower Partial Moment (SLPM) For some quantile $q<0$,

$$
\operatorname{SLPM}(q)=\int_{-\infty}^{q}(q-x)^{2} f(x) d x=2 \int_{-\infty}^{q}(q-x) F(x) d x
$$

First Lower Partial Moment (FLPM) For some quantile $q<0$,

$$
\operatorname{FLPM}(q)=\int_{-\infty}^{q}(q-x) f(x) d x=\int_{-\infty}^{q} F(x) d x
$$

Zeroth Lower Partial Moment (ZLPM) For some quantile $q<0$,

$$
\operatorname{ZLPM}(q)=\int_{-\infty}^{q} f(x) d x=F(q)
$$


Value-at-Risk (VaR) If $F(q)$ is fixed at $p$, then the inverse of the ZLPM gives VaR as

$$
\operatorname{VaR}(p)=-F^{-1}(p)=-q \text { where } q<0
$$

$\operatorname{VaR}(p)$ is the maximum potential loss to an investment with a prespecified confidence level $(1-p)$.

Expected Shortfall (ES) When the return distribution is continuous, ES at confidence level $(1-p)$ is defined as

$$
\begin{aligned}
\operatorname{ES}(q) & =-\mathrm{E}(x \mid x \leq q) \\
& =-\int_{-\infty}^{q} x \frac{f(x)}{F(q)} d x \\
& =-q+\frac{\operatorname{FLPM}(q)}{F(q)} \text { where } q<0 \text { and } F(q)=p
\end{aligned}
$$

Proposition 1 If the asset return distribution is heavy tailed with tail index $\alpha>0$ and scale coefficient $A>0$, then for $q<0$ the downside risk measures can be approximated as follows:

1. $\operatorname{SLPM}(q) \approx \frac{2 A(-q)^{2-\alpha}}{(\alpha-1)(\alpha-2)}, \alpha>2$

2. $\operatorname{FLPM}(q) \approx \frac{A(-q)^{1-\alpha}}{\alpha-1}, \alpha>1$

3. $\operatorname{ZLPM}(q) \approx A(-q)^{-\alpha}, \alpha>0$

4. $\operatorname{VaR}(p) \approx\left(\frac{A}{p}\right)^{\frac{1}{\alpha}}$ where $F(q)=p$

5. $\operatorname{ES}(q) \approx \frac{\alpha}{\alpha-1}\left(\frac{A}{p}\right)^{\frac{1}{\alpha}}, \alpha>1$

Proof. See Appendix A.

\subsection{Ordering Risk Measures}

Suppose that asset returns $X$ and $Y$ have regularly varying tails with tail indexes $\alpha_{1}>0$ and $\alpha_{2}>0$ and scale coefficients $A_{1}>0$ and $A_{2}>0$ respectively. Suppose that $\alpha_{1} \neq \alpha_{2}$ but $A_{1}=A_{2}$. In this case the asset return with lower tail index is more heavy tailed, and hence more risky than the asset with higher value of the tail index. 
Proposition 2 If $\alpha_{1}>\alpha_{2}$ and $A_{1}=A_{2}$, then the following relationships hold.

1. $\operatorname{SLPM}_{X}(q)<\operatorname{SLPM}_{Y}(q)$ for $\alpha_{1}>2, \alpha_{2}>2$

2. $\operatorname{FLPM}_{X}(q)<\operatorname{FLPM}_{Y}(q)$ for $\alpha_{1}>1, \alpha_{2}>1$

3. $\operatorname{ZLPM}_{X}(q)<\operatorname{ZLPM}_{Y}(q)$

4. $\operatorname{VaR}_{X}(p)<\operatorname{VaR}_{Y}(p)$

5. $\operatorname{ES}_{X}(q)<\operatorname{ES}_{Y}(q)$ for $\alpha_{1}>1, \alpha_{2}>1$

Proof. Differentiating the expressions for the downside risk measures as in Proposition 1, it follows that each downside risk measure is decreasing in $\alpha$. Hence the result.

Even when $A_{1} \neq A_{2}$, for $q<<0$, the above rankings will hold for $\alpha_{1}>\alpha_{2}$. This is because, in the expressions in Proposition 1, the coefficient $A$ appears in a linear manner while $-\alpha$ appears as a power of $-q$. Therefore, for $q<<0, \alpha$ will have a more dominating influence on the ranking of assets than $A$. Thus, going far enough out in the tail will make the asset ranking consistent with the fact that $\alpha_{1}>\alpha_{2}$, regardless of whether or not $A_{1}=A_{2}$. We state this as a corollary: ${ }^{2}$

Corollary 1 Suppose that asset returns $X$ and $Y$ have regularly varying tails with tail indexes $\alpha_{1}$ and $\alpha_{2}$ and scale coefficients $A_{1}$ and $A_{2}$ respectively. Suppose that $\alpha_{1}>\alpha_{2}$ and $A_{1} \neq A_{2}$. In this case for large loss levels, $q$, eventually the risk measures will rank the asset return with the lower tail index as more risky.

The Corollary means that the risk measures rank the two alternatives eventually lexicographically over $(\alpha, A)$.

Now, suppose that $\alpha_{1}=\alpha_{2}$ but the scale coefficients $A_{1} \neq A_{2}$. Without loss of generality, let $A_{1}<A_{2}$. In this case $F_{X}(-x)<F_{Y}(-x)$, hence $Y$ is more risky than $X$.

Proposition 3 If $\alpha_{1}=\alpha_{2}$ but $A_{1}<A_{2}$, then for large $q$, the following relationships hold.

$$
\text { 1. } \operatorname{SLPM}_{X}(q)<\operatorname{SLPM}_{Y}(q)
$$

\footnotetext{
${ }^{2}$ We are grateful to the referee for suggesting this corollary.
} 
2. $\operatorname{FLPM}_{X}(q)<\operatorname{FLPM}_{Y}(q)$

3. $\operatorname{ZLPM}_{X}(q)<\operatorname{ZLPM}_{Y}(q)$

4. $\operatorname{VaR}_{X}(p)<\operatorname{VaR}_{Y}(p)$

5. $\operatorname{ES}_{X}(q)<\operatorname{ES}_{Y}(q)$

Proof. Above can be proved easily by using the results from the Proposition 1.

Thus, all the risk measures give consistent ordering of the assets $X$ and $Y$ in this case.

Propositions 2 and 3 imply that in the tail region, we can order $X$ and $Y$ in a clear manner with respect to each of the downside risk measures. The ordering is consistent with the assumption that $X$ is less risky than $Y$. The similar preference ordering of risk, as observed above, is in line with the empirical findings of Hahn et al. (2002). Using data from the trading book of an investment bank, they found empirically that many of the downside risk measures, including those considered here, assess risk of the trading portfolios in nearly the same way. Propositions 2 and 3 explain this similarity in an analytical manner.

\section{Conclusion}

We examine downside risk measures for heavy tailed distributions defined as distributions with regularly varying tails. Using tail approximations of regularly varying tails, we provide expressions that approximate the various downside risk measures as functions of the tail coefficient and scale index. We show that all downside risk measures provide preference ordering consistent with the notion of risk conveyed by the tail indexes and the scale coefficients of heavy tailed assets. Thus, for heavy tailed distributions, choice of downside risk measures does not seem to matter much as all downside risk measures order heavy tailed risk in a similar manner. 


\section{Appendix A: Derivation of the Expressions in Proposition 1}

Suppose that the distribution of $X$ has a regularly varying tail. Then, to a first order approximation,

$$
F(-x) \approx A x^{-\alpha}, \text { as } x \rightarrow \infty \text { where } A>0, \alpha>0
$$

1. $\operatorname{SLPM}(q)$

$$
\begin{aligned}
\operatorname{SLPM}(q) & =2 \int_{-\infty}^{q}(q-x) F(x) d x, q<0 \\
& \approx 2 \int_{-\infty}^{q}(q-x) A(-x)^{-\alpha} d x \\
& =2 \int_{-q}^{\infty}(q+y) A y^{-\alpha} d y \text { where } y=-x \\
& =2 q A\left|\frac{y^{1-\alpha}}{1-\alpha}\right|_{-q}^{\infty}+2 A\left|\frac{y^{2-\alpha}}{2-\alpha}\right|_{-q}^{\infty} \alpha>2 \\
& =\frac{2 A(-q)^{2-\alpha}}{(\alpha-1)(\alpha-2)}, \alpha>2
\end{aligned}
$$

2. $\operatorname{FLPM}(q)$

$$
\begin{aligned}
\operatorname{FLPM}(q) & =\int_{-\infty}^{q} F(x) d x \\
& \approx \int_{-\infty}^{q} A(-x)^{-\alpha} d x \\
& =\int_{-q}^{\infty} A y^{-\alpha} d y \text { where } y=-x \\
& =\frac{A(-q)^{1-\alpha}}{\alpha-1}, \alpha>1
\end{aligned}
$$

3. $\operatorname{ZLPM}(q)$

$$
\begin{aligned}
\operatorname{ZLPM}(q) & =F(q) \\
& \approx A(-q)^{-\alpha}
\end{aligned}
$$


4. $\operatorname{VaR}(p)$

$$
\begin{aligned}
F(q) & =p \\
p & \approx A(-q)^{-\alpha} \\
\operatorname{VaR}(p)=-q & \approx\left(\frac{A}{p}\right)^{\frac{1}{\alpha}}
\end{aligned}
$$

5. $\operatorname{ES}(q)$

$$
\begin{aligned}
\operatorname{ES}(q(p)) & =-q+\frac{\operatorname{FLPM}(q)}{F(q)} \\
& \approx-q+\frac{A(-q)^{1-\alpha}}{A(-q)^{-\alpha}(\alpha-1)} \\
& =\frac{\alpha}{\alpha-1}(-q) \\
& =\frac{\alpha}{\alpha-1}\left(\frac{A}{p}\right)^{\frac{1}{\alpha}}
\end{aligned}
$$




\section{References}

Bawa, V. S. (1975). Optimal rules for ordering uncertain prospects. Journal of Financial Economics, 2(1):95-121.

Bingham, N. H., Goldie, C. M., and Teugels, J. L. (1987). Regular variation. Cambridge University Press, Cambridge.

de Haan, L., Resnick, S. I., Rootzen, H., and de Vries, C. G. (1989). Extremal behaviour of solutions to a stochastic difference equation with applications to arch-processes. Stochastic Processes and their Applications, pages 213224 .

Dhaene, J., Goovaerts, M. J., and Kaas, R. (2003). Economic capital allocation derived from risk measures. North American Actuarial Journal, $7(2): 44-56$.

Engle, R. (1982). Autoregressive conditional heteroscedasticity with estimates of the variance of United Kingdom inflation. Econometrica, 50:9871007.

Fishburn, P. C. (1977). Mean-risk analysis with risk associated with below target returns. American Economic Review, 67(2):116-126.

Hahn, C., Pfingsten, A., and Wagner, P. (2002). An empirical investigation of the rank correlation between different risk measures. Westfalische Wilhelms-Universitat Munster working paper.

Jansen, D. W. and de Vries, C. G. (1991). On the frequency of large stock returns: Putting booms and busts into perspective. The Review of Economics and Statistics, 73(1):18-24.

Mandelbrot, B. B. (1963). The variation of certain speculative prices. Journal of Business, 36:392 - 417.

Pagan, A. (1996). The econometrics of financial markets. Journal of Empirical Finance, 3:15 - 102.

Resnick, S. I. (1987). Extreme values, regular variation and point process. Springer-Verlag.

Roy, A. D. (1952). Safety first and the holding of assets. Econometrica, 20(3):431-449. 